\title{
Design and Study of Intelligent Cutting Machine for Shelling of Camellia Fruit
}

\author{
Kaiwu Cai ${ }^{a}$, Wei Cheng ${ }^{\mathrm{b},{ }^{*}}$ and Xuanping Yang ${ }^{\mathrm{c}}$ \\ Huali College Guangdong University of Technology, Guangzhou 511325, China. \\ a171121932@qq.com, bchengwei3530@163.com, cyangxuanping0907@qq.com
}

Keywords: Camellia fruit, Shell, machine design, PRO/E.

\begin{abstract}
China Camellia fruit shell to mechanical machine has not been popular, especially in rural areas, this paper designs a kind of advanced and applicable Camellia fruit shell device, used to make more efficient fruit tea, Camellia fruit hulling machine, which comprises a shell, a cutting mechanism, tie breaking mechanism and a driving piece, the parameters of the design by PRO/E software for three-dimensional modeling, simulation of the 3D effect, through rational design of motion simulation movement principle, and then obtain the optimal design parameters by software, so as to optimize the design, implementation to the same direction to ensure complete separation of the crushed shells, while avoiding damage to the seeds. In the extrusion process, can save a lot of labor cost, the whole structure is simple, easy to implement, can be applied to.
\end{abstract}

\section{Introduction}

Camellia fruit is a unique woody oil resource in China. The fatty acid composition of camellia oil is similar to olive oil. It is known as "Oriental olive oil", "king of oil" and "gold liquid". Camellia oil contains essential monounsaturated fatty acids as high as $90 \%$, rich in vitamin E, tea polyphenols, tea camellia glycosides, saponins, and calcium, iron, zinc and other elements and antioxidant VE and squalene, is a kind of woody plants also have edible value, medicinal value and health value of oil [1-2].

The pickled Camellia fruit needs to be processed to crack the shell, remove the shell and get the fruit seed, and then press it. The traditional method of separation is the camellia fruit exposure, the shell cracking seeds from natural shedding, the method has the need to spend a lot of manpower, shelling is not complete, the efficiency is low, not suitable for mass production and other issues, and the restriction of weather is large, is not conducive to stable production. In order to overcome the shortcomings of the existing technology, this paper studies a high efficiency and thorough shelling machine.

\section{The Design of the Shell Removing Machine}

The analysis of the shape of Camellia fruit is spherical, and a square has six sides and eight vertices. According to the idea of the camellia fruit cut into a approximate cube shape, and then the eight vertices can be removed after the shell of Camellia seed.

The working principle of the scheme is as shown in Fig 1. 


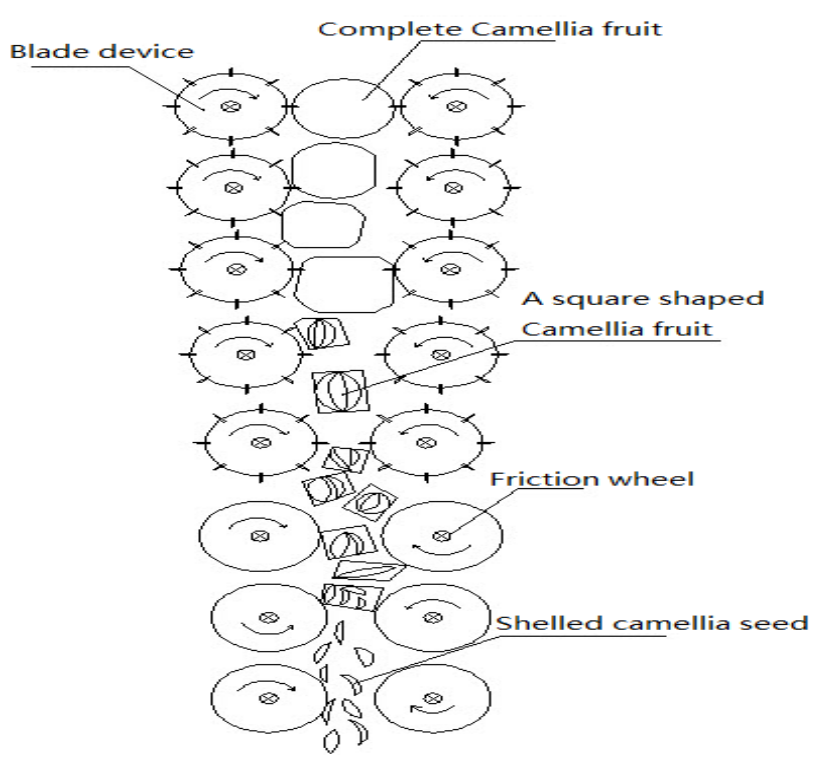

Fig 1 Working principle diagram

The long strip blade is used to install the long strip blade on the axis, and the turning on the left and right sides of the axis is opposite. The left and right sides of the shaft to form a " $V$ " shape, because the camellia fruit will gradually be cut into approximate cube, volume becomes smaller, so the use of "V" shape. By cutting into the approximate cube of the camellia fruit continue to be at the lower end of the three round of friction, the friction is designed for preventing friction when the hands back and forth, on both sides of the friction wheel for a clockwise rotation, a clockwise rotation, when the camellia fruit after being cut through the friction wheel at that moment was an upward and a downward force on both sides, so as to achieve the effect of friction on the purpose of Camellia fruit, friction is not cut off the shell by relative movement will be the removal [3].

\section{Design of Transmission Part}

The main transmission system of this device is belt drive and gear transmission. The power is produced by Shenyang Hongda Motor Manufacturing Co., Ltd., which has a motor of 56. This kind of motor has the characteristics of versatility, small volume, large output power, reliable structure, beautiful appearance and so on. [4] Because the belt drive directly transfers the power of the motor to the power system, so the belt speed should be in the range of $5 \sim 25 \mathrm{~m} / \mathrm{s}$. If we fail to reach the high speed motor, we should reduce the speed of the motor through the decelerator.

For the design of belt drive, the belt drive has friction belt drive and meshing belt drive. The friction type belt wheel transmission according to the cross section shape is rectangular, trapezoidal or round, can be divided into flat belt, $\mathrm{V}$ belt, and wedge belt drive and round belt drive. The device uses a friction type $\mathrm{V}$ belt to drive [5].

The transmission of the motor to the friction wheel is selected to design the $\mathrm{V}$ pulley, and the $\mathrm{V}$ pulley here is a single $\mathrm{V}$ belt drive, as shown in Fig 2.

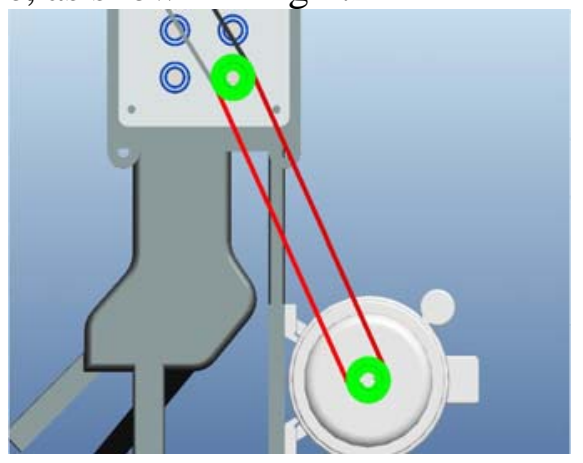

Fig 2 Single V belt drive 
For the design of the shaft, the axis of the loading tool is designed. The design axis needs to choose the right material. The material of the shaft is carbon steel (35, 45, 50 and other high quality medium carbon steel). Because the strength requirement of the shaft is relatively small, the ordinary carbon steel such as Q237 or Q275 can meet the requirements. In this device, the grooves that can be loaded on the shaft are designed on the shaft, and the staircase is designed.

The power transmission system of the sheller is greatly different from the power transmission system of the common device. Because the steering of the left and right axes of the loader is opposite, it also ensures that the other axis of the same side has the same direction of rotation. So in the first on the other end of the cutter shaft gear cleverly designed for the direction of rotation axis of a shaft clockwise, a shaft counterclockwise, and both sides of Camellia fruit for cutting, the right side of the tool drives the first axis counterclockwise rotation through the rotation direction of the gear transmission. The tool belt drives the same side with the rest of the same, and the lower left side of the friction wheel cutter and cutter with drive through the rest of the same side with the same direction of rotation [6]. The three-dimensional effect of this part of the structure is shown in Fig.3 and Fig.4.

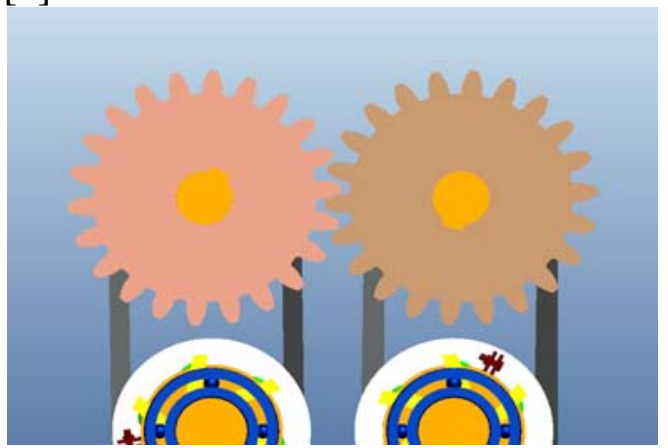

Fig.3 Gear transmission at the cutting tool

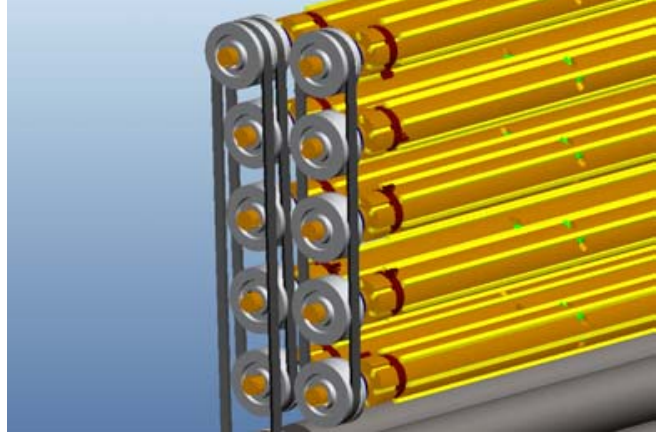

Fig.4 Belt drive at the cutting tool

\section{Cutting Part Design}

This part is mainly composed of the blade cutting the camellia fruit and the friction wheel to remove the excess shell. The number of axial loading blade according to the cutting process and Camellia fruit is selected. Using three dimensional software "PRO/E" to assist the design of the sheller [7], the main cutting process, such as Fig 5
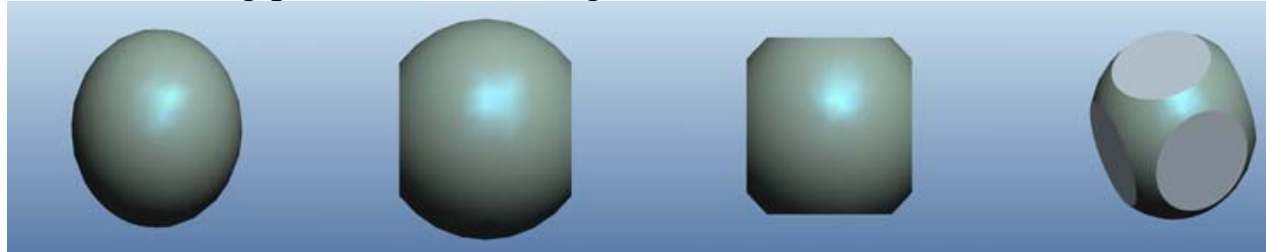

Fig.5 The cutting process of Camellia fruit

Complete the fruit is cut into cubes at least approximate Camellia use three knife device, the device fully considering cutting, five knife device and friction device for removing eight vertices of the set for three. This part is a device for obtaining camellia seed by removing the newly picked Camellia fruit directly from the shell.

\section{Design of Separation Mechanism}

When the fruit shell camellia, camellia seed and shell needs to be separated from the chip, so to design a similar selection mechanism to get clean Camellia seed. As the shell volume is much smaller than the camellia seed, a simple separation structure is designed for this characteristic, and its structure diagram is shown in Fig.6. 


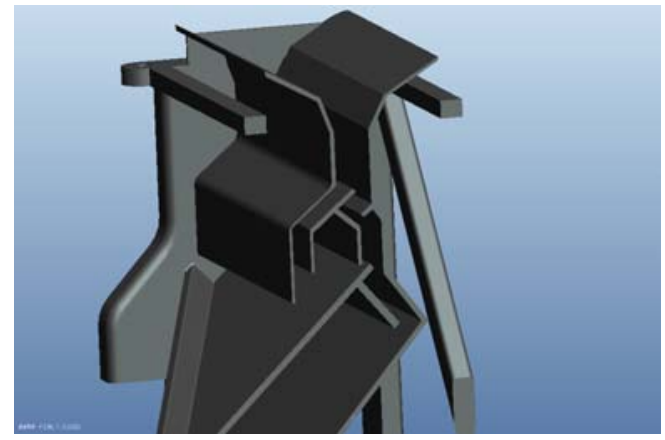

Fig.6 The internal three-dimensional effect diagram of the separation mechanism

\section{Whole Machine Dynamics Analysis}

Through the previous design process, we use the three-dimensional software PRO/E to make three-dimensional modeling for the design plan, and get the assembly diagram of the cutter device, friction device, cutter device and friction device as shown in Fig.7:

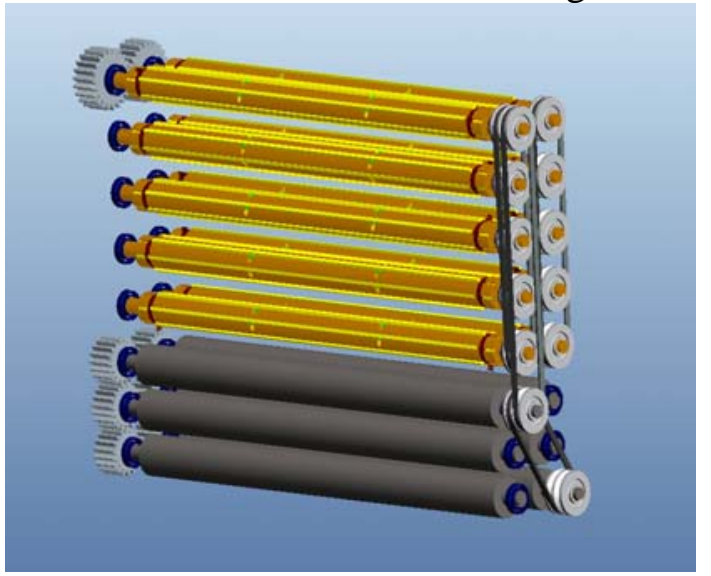

Fig.7 Assembly of blade and friction device

After designing the whole machine, we need to simulate the whole working principle and test the whole machine so as to find out the design flaws in time and simulate the whole machine with the help of the three-dimensional design software "PRO/E".

The first is the various parts of the design of the assembly in accordance with assembly relationship correctly, after assembly need to add virtual device to provide power to the motor, according to the principle described above to establish virtual belt transmission belt transmission, the establishment of virtual gear on the gear, such as the establishment of virtual environment movement, add right for these conditions and constraints was as shown in Fig.8 and Fig.9.

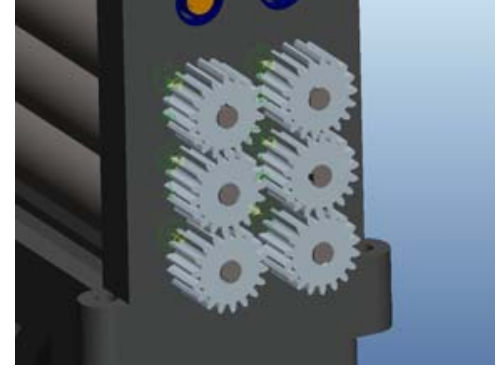

Fig.8 Link to Gear Loading

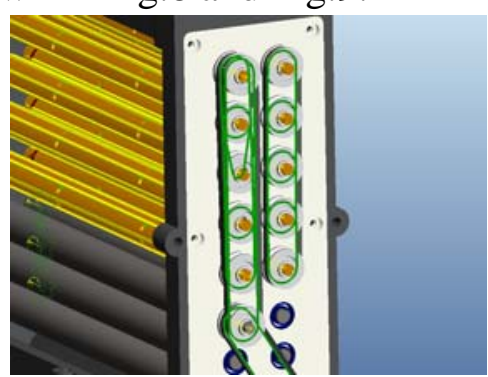

Fig.9 And wheel load link

The speed, acceleration and other parameters of the components of the transmission system can be measured by the software, and the speed of the motor to the friction wheel is measured. 


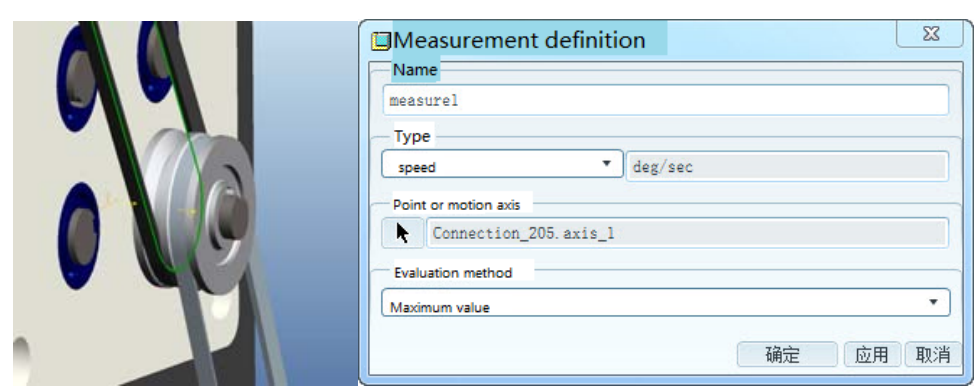

Fig.10 Measurement objects and measurement definitions

The measuring object is the maximum speed of the motor transmitted to the friction wheel, and its curve is shown in Fig.11.

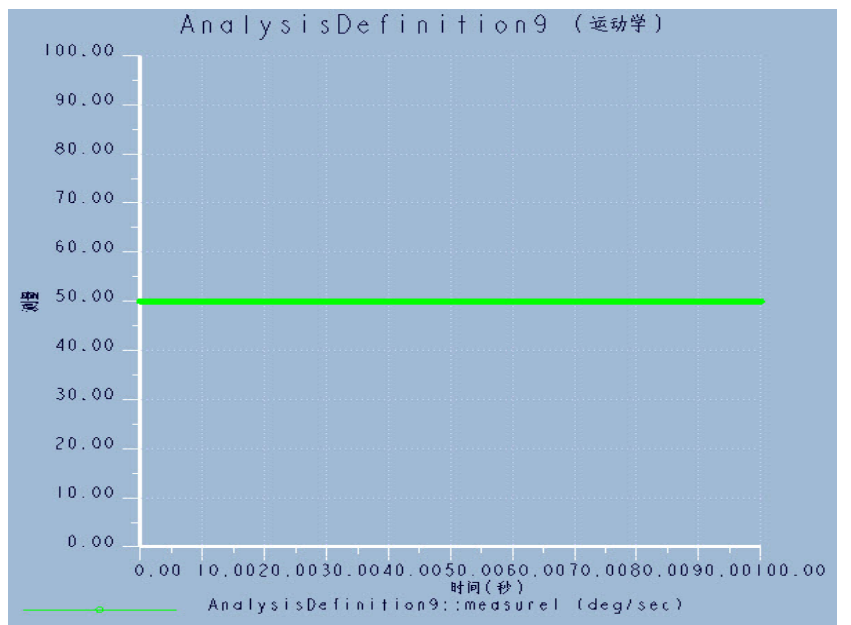

Fig.11 Curve of measurement results (unit deg/sec)

The maximum speed of the motor to be transmitted to the friction wheel from Fig. 11 is $50 \mathrm{deg} / \mathrm{sec}$. The final structure diagram of the shell removal machine is shown in Fig.12.
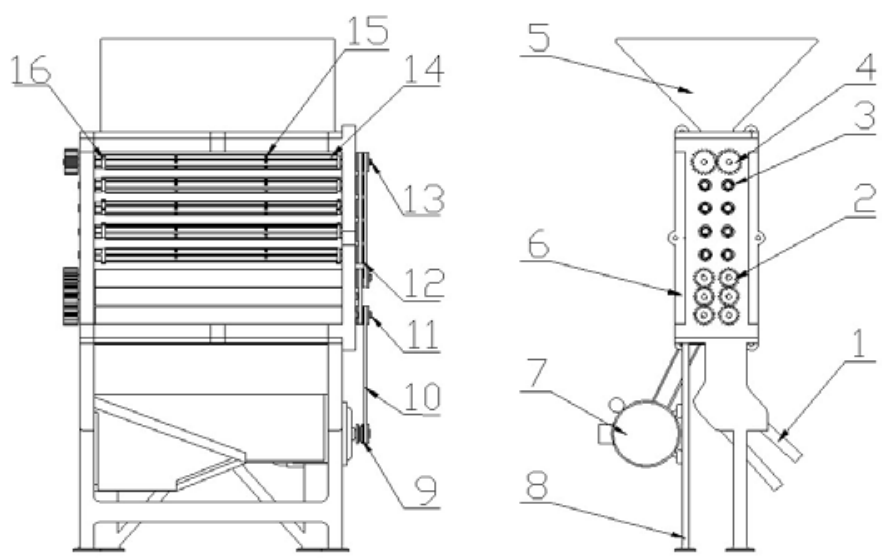

1. landing gear; 2. friction shaft gear; 3. bearing; 4. gear; 5. hopper; 6. rack; 7. motor; 8. support frame; 9. double belt pulley; $10 . \mathrm{V}$ belt; 11 . friction shaft; 12 . pulley; 13. long blade shaft; 14 . long blade; 15. pin; 16. clamping sleeve. Summary

Fig.12 The structure of the sheller

The small family shelling device for rural areas should not only ensure the efficiency of shell removal, but also ensure small volume, simple operation and favorable price. It directly shuck the harvested Camellia fruits and obtain camellia seeds. In this paper, a design of high efficiency and thorough shelling Camellia fruit sheller, and virtual operation, design of the machine motion simulation, dynamic analysis, stress analysis, breaking the traditional processing procedures, this machine has applied for invention patent, can save a lot of labor costs, the whole body has the advantages of simple structure, convenient operation. Can be applied to the market, good prospects. 


\section{Acknowledgments}

This project is supported by Foundation for Comprehensive reform of mechanical design and manufacturing and automation (Cantonese high school letter [2014]97). Innovation ability training experimentation area of mechanical and electrical specialty (Cantonese high school letter [2015]133). The Youth Innovative Talent Project of Guangdong general colleges and universities in 2018. Key cultivation discipline of innovation and strong school in Guangdong Province in 2017 Mechanical engineering and automation.

\section{References}

[1]. Li Li, Xuehui Wu, Qiaohua Kou. Research status and application prospect of tea oil [J]. Chinese oil. (2010) No. 3, p. 10-14.

[2]. Yongzhong Chen, Jian Luo, Rui Wang. The present situation and Prospect of the development of Chinese oil tea industry [J]. Grain science and technology and economy. (2013 )No.1, p. 10-12.

[3]. Rensheng Guo. Mechanical engineering design analysis and MATLAB application [M]. Beijing: Machinery Industry Press, 2008, p. 123-135.

[4]. Feng Zhang, Zhibin Yang, Jingfu Zhang. The design principle of Camellia fruit peeling machine mechanical [J]. Hubei forestry science and technology. (2013) No.3, p. 21-23.

[5]. Fenghong Huang, Wenlin Li, Fujian Xia. Development and application of agricultural engineering [J]. Journal of camellia seed sheller. (2006) No.11, p. 147-151.

[6]. Jing Xiao; Lijun Li .Design and Experimental Research on gripper type oil tea fruit shell seed cleaning machine; Research on mechanization of agricultural mechanization [J]. (2017) No.4, p.93-97.

[7]. Jianjun Qiao, Baoping Wang, Renxi Hu.Proe/ENGINEER Wildfire 5 dynamics and finite element analysis from entry to master [M]. Beijing: Machinery Industry Press, 2010, p. 53-70. 